\title{
Physical activity counteracts tumor cell growth in colon carcinoma C26-injected muscles: an interim report
}

\author{
Charlotte Hiroux, Tijs Vandoorne, Katrien Koppo, Stefan De Smet, Peter Hespel, Emanuele \\ Berardi \\ Exercise Physiology Research Group, Department of Kinesiology, KU Leuven, Belgium \\ This article is distributed under the terms of the Creative Commons Attribution Noncommercial License (CC BY-NC 4.0) which \\ permits any noncommercial use, distribution, and reproduction in any medium, provided the original author(s) and source are credited.
}

\begin{abstract}
Skeletal muscle tissue is a rare site of tumor metastasis but is the main target of the degenerative processes occurring in cancer-associated cachexia syndrome. Beneficial effects of physical activity in counteracting cancer-related muscle wasting have been described in the last decades. Recently it has been shown that, in tumor xeno-transplanted mouse models, physical activity is able to directly affect tumor growth by modulating inflammatory responses in the tumor mass microenvironment. Here, we investigated the effect of physical activity on tumor cell growth in colon carcinoma C26 cells injected tibialis anterior muscles of BALB/c mice. Histological analyses revealed that 4 days of voluntary wheel running significantly counteracts tumor cell growth in C26-injected muscles compared to the non-injected sedentary controls. Since striated skeletal muscle tissue is the site of voluntary contraction, our results confirm that physical activity can also directly counteract tumor cell growth in a metabolically active tissue that is usually not a target for metastasis.
\end{abstract}

Key Words: cancer cachexia, colon carcinoma C26 cells, tumor cell growth, muscle inflammation, voluntary running

Eur J Transl Myol 2016; 26 (2): 67-75

Cancer cachexia is a multifactorial catabolic syndrome associated with tumor progression. ${ }^{1}$ It is characterized by progressive muscle degeneration mediated by an imbalance between protein synthesis and protein degradation in favor of an increased rate of skeletal muscle proteolysis. ${ }^{2-4}$ This chronic wasting condition is mainly sustained by high levels of circulating proinflammatory cytokines and tumor-released factors $(e . g$. interleukins $1 \beta$ and 6 (IL1 $\beta$, IL6), tumor necrosis factor alpha (TNF- $\alpha$ ) and proteolysis inducing factor (PIF)), which specifically trigger the proteolysis of muscle proteins. ${ }^{5}$ To date, no specific cures exist for cancer cachexia and the pharmacological strategies commonly adopted aim to treat the cancer itself, rather than targeting degenerative molecular mechanisms at the muscle site. ${ }^{4}$

Recently, there is an emerging interest in the possible effects of physical activity on the incidence, prognosis and treatment of cancer. ${ }^{6-8} \mathrm{~A}$ wide range of beneficial effects mediated by physical exercise in cancer cachexia have been reported in both murine ${ }^{9}$ and human studies. $^{7,8,10}$ Epidemiological studies showed that physical activity has a pivotal role in reducing tumor incidence, ${ }^{6,11}$ improving responsiveness to chemotherapy ${ }^{8}$ and, in general, ameliorating cancer patients' quality of life. ${ }^{11-13}$ In this view, physical exercise has been proposed as an adjuvant therapy in cancer, including breast ${ }^{11,14}$ and colon cancers. ${ }^{11}$

At the molecular level, physical exercise has been identified as a key modulator of systemic inflammation $^{12}$, able to counteract muscle atrophy by restoring the physiological autophagic flux, to decrease muscle proteolysis and to preserve muscle mass and function in both cancer patients $7,8,10,12,13,15$ and tumorbearing mice. ${ }^{9,16}$ A recent report described a causal relationship between physical activity and tumor growth inhibition. ${ }^{17}$ Pedersen et al. ${ }^{17}$ showed that voluntary running suppresses tumor growth by remodeling the inflammatory background in the tumor microenvironment in several tumor models. Specifically, high circulating epinephrine levels due to voluntary running increase NK cell tumor infiltration which in turn impairs tumor growth. Although many studies address the beneficial effects of physical 


\section{Physical activity counteracts tumor cell growth in muscle}

Eur J Transl Myol 26 (2): 67-75

Table 1. Physical activity. Values are means \pm SEM of 3 independent experiments per group

\begin{tabular}{|l|c|c|c|c|}
\hline \multirow{2}{*}{} & \multirow{2}{*}{$\begin{array}{c}\text { Total DST } \\
(\mathrm{Km})\end{array}$} & \multicolumn{2}{|c|}{ Speed at day $4(\mathrm{Km} / \mathrm{h})$} & \multirow{2}{*}{$\begin{array}{c}\text { Average of total time } \\
(\mathrm{hh}: \mathrm{mm}: \mathrm{ss})\end{array}$} \\
\cline { 3 - 4 } & & Average & Max & \\
\hline Ctr WR & $18.06 \pm 3.52$ & $1.3 \pm 0.07$ & $2.5 \pm 0.09$ & $15: 57: 47 \pm 02: 17: 12$ \\
\hline C26 WR & $19.55 \pm 2.60$ & $1.4 \pm 0.06$ & $2.5 \pm 0.04$ & $14: 55: 29 \pm 01: 54: 17$ \\
\hline
\end{tabular}

exercise at multi-organ and multi-systemic levels, ${ }^{18}$ it is still not clear why skeletal muscle tissue, which is a main tissue target of pathophysiological changes occurring in cancer cachexia, ${ }^{2,5}$ is refractory to tumor cell colonization and metastasis. ${ }^{19-21}$

Here, we performed histological analyses of tibialis anterior (TA) muscles from mice injected with colon carcinoma C26 cells to test the effects of physical activity on tumor cell growth specifically at the contraction site. Our results showed a significant reduction of tumor cell growth in muscles from physically active mice compared with the sedentary ones. These data confirm the ability of physical exercise to counteract tumor cell growth.

\section{Materials and Methods}

\section{Mice}

Nine 8-weeks-old male BALB/c mice were used for this study. To study the effects of physical activity on tumor cell growth, $1 \times 10^{4}$ murine colon carcinoma C26 cells were re-suspended in PBS $(50 \mu 1)$ and injected in tibialis anterior (TA) muscles. TA from the contralateral leg was injected with the same volume of PBS as control in each mouse. Mice were housed in standard conditions with day/night cycles of 12 hours, received water and food ad libitum, and were euthanized 4 days after tumor injection.

All the mice used in this study were treated in accordance with ARRIVE guidelines and following the three R's rule of Replacement, Reduction and Refinement principles. ${ }^{22}$ Protocols adopted in the study have been approved by the animal experimentation ethics committee of KU Leuven, Belgium.

\section{Exercise protocol}

Mice were randomly assigned to three experimental groups: (i) C26-injected mice hosted in standard cages (sedentary control group, C26 REST), (ii) non-injected running mice hosted in wheel-equipped cages (running control group, Ctr WR) and (iii) C26-injected running mice hosted in wheel-equipped cages (C26 WR). Cages were prepared as previously described. ${ }^{23}$ Briefly, one cage per mouse was used and all wheels were supplied with a cycle computer in order to record physical activity data (i.e. daily distance, total distance, average and maximum speed and time spent on the wheel). Mice in the running groups were hosted in wheel-equipped cages starting from the day before tumor cell injection, to familiarize the animals with the use of the new environmental stimulus (i.e. wheel) until the day of sacrifice (4 days after tumor cells injection).

\section{Histology}

TA muscles were dissected, weighed, embedded in tissue freezing medium (Leica, Wetzlar, GE), frozen in liquid nitrogen-cooled isopentane and stored at $-80^{\circ} \mathrm{C}$. Muscle cryosections of $7 \mu \mathrm{m}$ thickness were obtained using a cryostat (Leica Biosystems). For histological analysis, the sections were stained with Hematoxylin and Eosin (H\&E, Sigma-Aldrich) using a standard method.
A

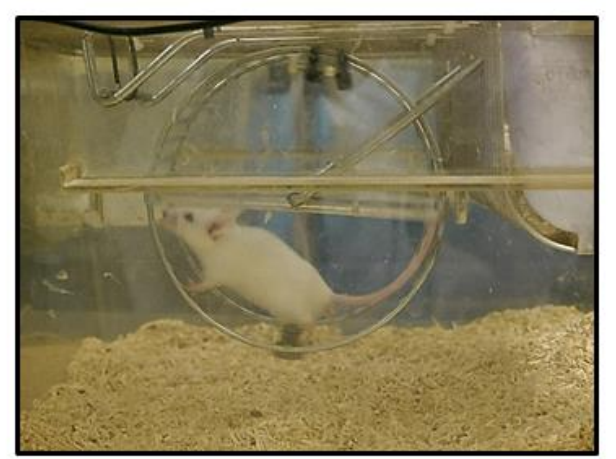

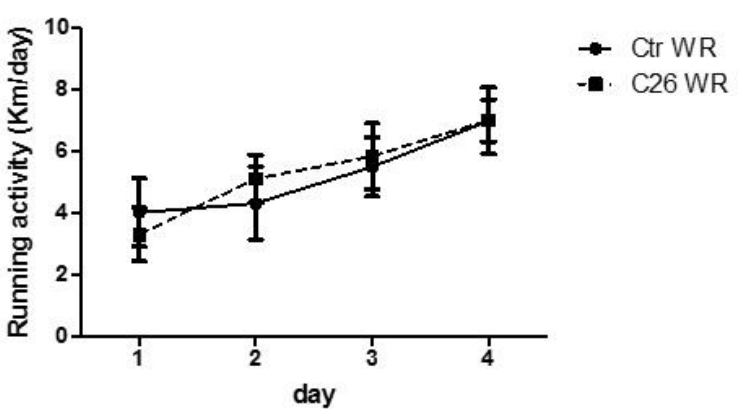

Fig 1. Voluntary wheel running exercise. (A) Mice from running groups were hosted in wheel-equipped cages. (B) non-injected running mice (Ctr WR) and C26-injected running mice (C26 WR) covered comparable daily distance until the end of experiment, 4 days. Data are means $\pm S E M$ of 3 independent experiments. 


\section{Physical activity counteracts tumor cell growth in muscle}

Eur J Transl Myol 26 (2): 67-75

Table 2. Body and muscle weight.

\begin{tabular}{|l|c|c|c|c|}
\hline \multirow{2}{*}{} & \multicolumn{2}{|c|}{ Body weight (BW) } & \multicolumn{2}{c|}{ Muscle weight/BW (mg/gr) } \\
\cline { 2 - 5 } & Initial & Final & C26 & PBS \\
\hline C26 REST & $26.47 \pm 0.97$ & $25.13 \pm 1.04$ & $2.96 \pm 0.06^{*}$ & $2.38 \pm 0.03$ \\
\hline C26 WR & $24.93 \pm 0.29$ & $23.17 \pm 0.27$ & $2.67 \pm 0.11$ & $2.43 \pm 0.09$ \\
\hline
\end{tabular}

Values are means \pm SEM of 3 independent experiments per group.

TA C26 REST versus TA PBS REST: *p < 0.05 by 1 way ANOVA.

\section{Immunofluorescence}

Transverse cryosections were fixed in $4 \%$ paraformaldehyde for $10 \mathrm{~min}$ at room temperature (RT) and permeabilized in $1 \%$ bovine serum albumin (BSA) $/ 0.2 \%$ Triton in Phosphate-Buffered Saline solution (PBS) for $30 \mathrm{~min}$. After incubation with $1 \%$ BSA in PBS (30 min), samples were incubated with a goat polyclonal anti-Ki67 Ab (Sc-78, Santa Cruz) (1:100 in BSA), followed by incubation with anti-goat Alexa fluor 488 conjugated $\mathrm{Ab}$ (Molecular Probes, Eugene, OR) (1:500 in BSA). Macrophage staining: samples were incubated with a rat anti-mouse F4/80 (Bio Rad MCA 497G), followed by incubation with anti-mouse Alexa fluor 594 conjugated Ab (Molecular Probes, Eugene, OR). IgG-immunostaining was performed by incubation with anti-mouse Alexa fluor 594 conjugated $\mathrm{Ab}$ (Molecular Probes, Eugene, OR) (1:500 in BSA) for $45 \mathrm{~min}$ at RT. Nuclei were stained for $5 \mathrm{~min}$ with 0.5 $\mu \mathrm{g} / \mathrm{ml}$ Hoechst 33342 (Sigma).

Morphometric analysis

Morphometric analysis was performed on pictures from H\&E staining. Three different cross-sectional areas of each muscle were analyzed. Muscle and tumor areas were calculated as relative percentages. ImageJ Software (National Institutes of Health, Bethesda, MD, USA) was used to perform this analysis.

\section{Statistics}

All quantitative data are presented as mean \pm standard error of mean (SEM) of three independent experiments. Statistical analysis was performed using Student's t-test or one-way and two-way ANOVA followed by Bonferroni post-hoc testing using GraphPad Prism 5 software (GraphPad Software, San Diego, CA). A p-value less than 0.05 was considered to be statistically significant

\section{Results}

Voluntary running activity showed no significant differences in terms of daily distance, total distance, average running speed and total running time (Fig. $1 \mathrm{~B}$ and Table 1) between non-injected and C26-injected mice. These data indicate that the amount of tumor cells injected in the muscles did not affect voluntary physical activity of the mice.

By histological analyses we evaluated the effects of
A

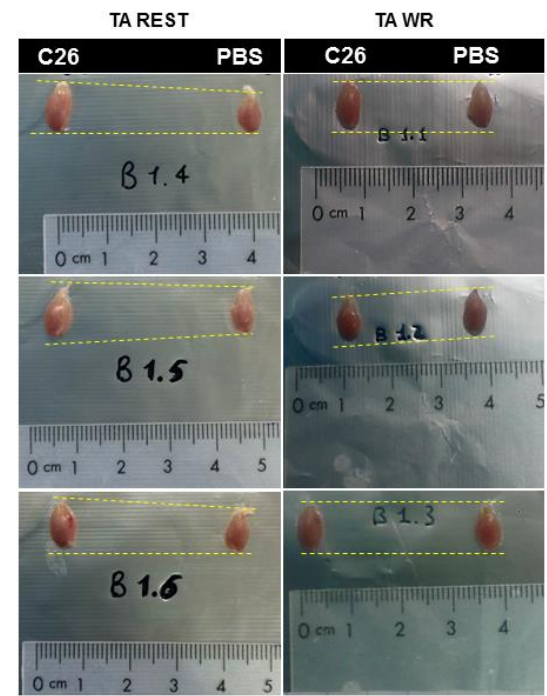

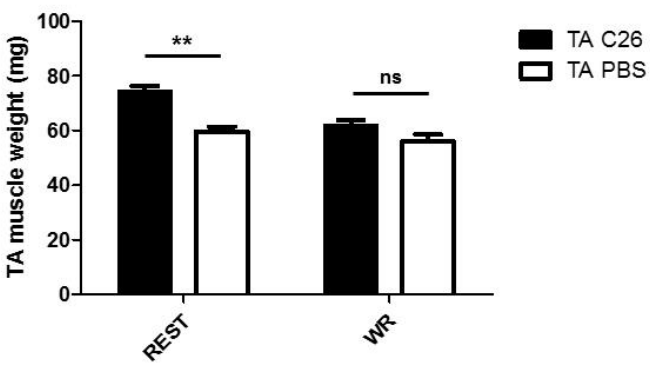

Fig 2. Size and muscle weight analyses. (A) In C26 REST mice (B1.4, B1.5 and B1.6) C26-injected TA muscles appear to be larger than the contralateral PBS-injected muscles compared with those derived from C26 WR (B1.1, B1.2 and B1.3). Dashed yellow lines help the comparisons. (B) Muscle weight analysis confirmed the differences observed in (A). Error bars are shown as means \pm SEM of 3 independent experiments; ** $p=0.01$ by 2 way ANOVA. 
Physical activity counteracts tumor cell growth in muscle Eur J Transl Myol 26 (2): 67-75

A

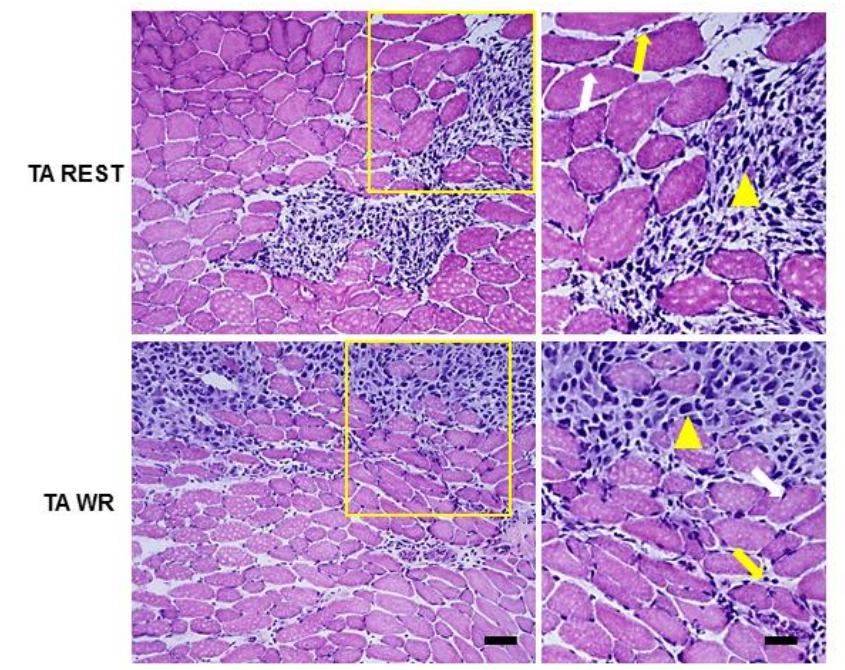

B
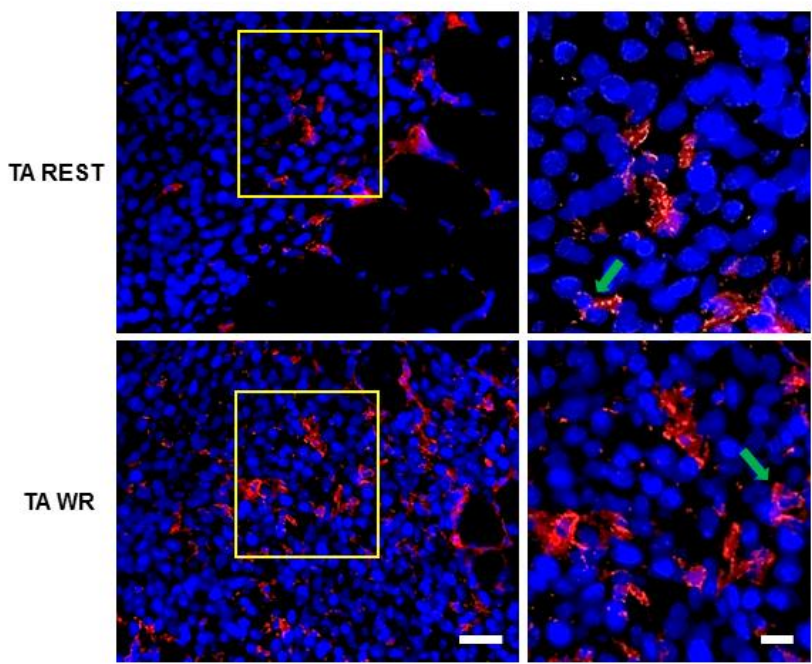

Fig 3. Histological features of areas containing tumor cells in C26-injected muscles. C26-injected muscles from mice at rest and running conditions developed tumor areas characterized by a high number of cells/area. Tumor cells had bigger nuclei (yellow arrowheads), compared with the infiltrating cells observed in the tumor areas (yellow arrows) or myonuclei (white arrows). Right up and down panels represents magnification areas from yellow rectangles of left panels. Bars: bottom left $=100 \mu \mathrm{m}$, bottom right $=200 \mu \mathrm{m}$.

physical activity on tumor growth 4 days after tumor cell injection in TA muscles from mice at rest and under voluntary running conditions. Since the study was performed using a small number of animals per experimental group $(n=3)$, we tracked samples from every mouse in order to display the consistency of the
A
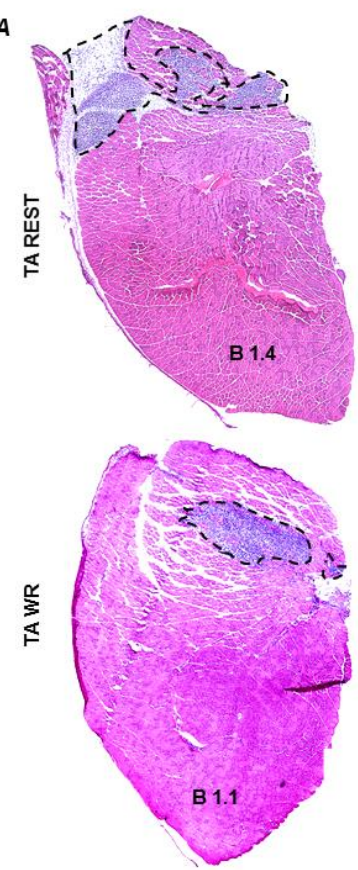
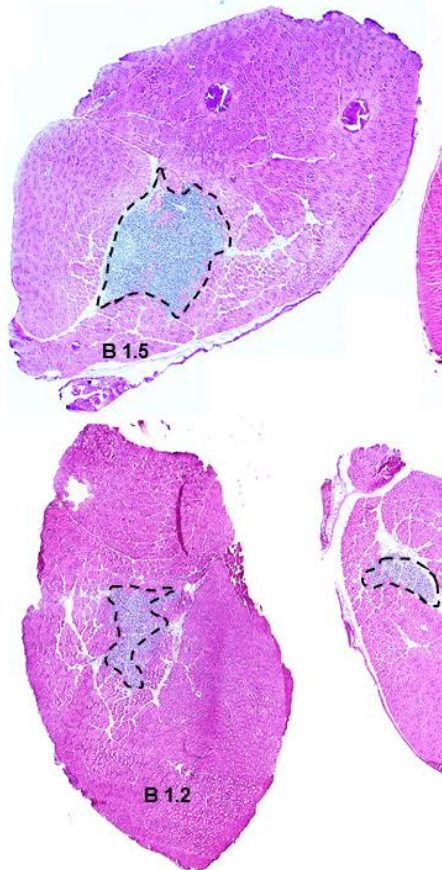

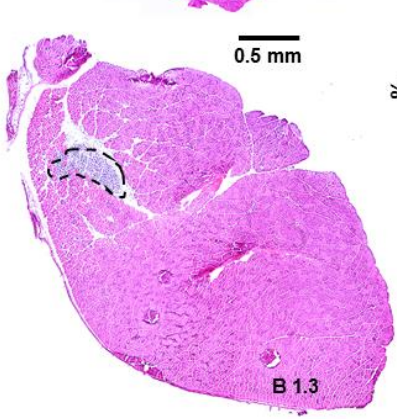

B 1.3

Fig 4. H\&E staining of C26-injected muscles. (A) H\&E staining of representative transverse cryosections obtained by photomicrographs reconstruction. Dashed black lines represents areas of tumor cell growth. (B) Morphometric analysis of tumor cell growth. Bar $=0.5 \mathrm{~mm}$. Error bars are shown as means \pm SEM of 3 independent experiments. 
A

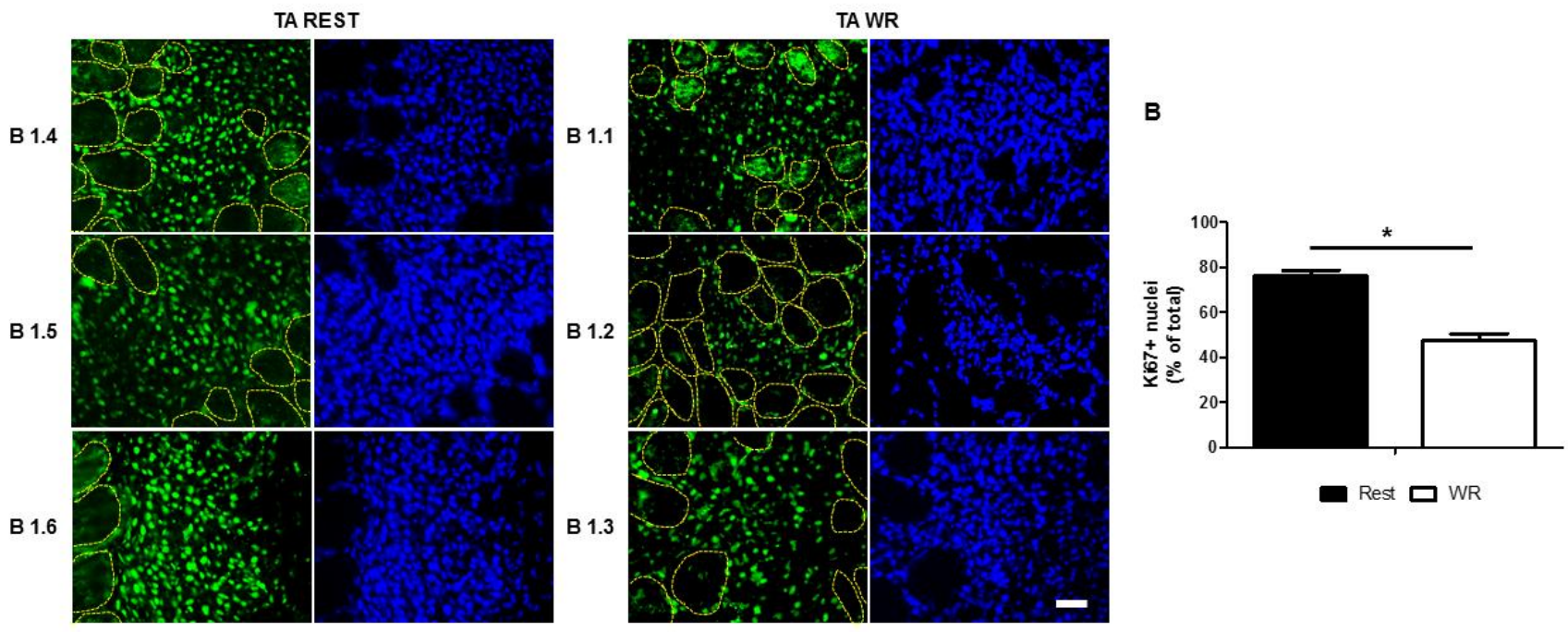

Fig 5. Tumor cell growth in C26-injected muscles. (A) Representative images of tumor cell growth areas. Green, Ki67 positive nuclei, dashed yellow lines represent muscle fibers, blue Hoechst. (B) Tumor cll growth analysis. Error bars are shown as means \pm SEM of 3 independent experiments; * $p<0.05$ compared with Student's t-test.

results. Specifically, mice from the sedentary injectedgroup (C26 REST) are indicated in the figures as B1.4, $\mathrm{B} 1.5$, B1.6, while mice from voluntary running injectedgroup (C26 WR) are indicated as B1.1, B1.2, and B1.3, according with the experimental recording system adopted in our laboratory. At the end-point (sacrifice day), C26-injected muscles from mice at rest were bigger than the PBS-injected contralateral ones, while no significant differences were found between C26 and
PBS-injected muscles from the exercised group (Fig. 2 A). Muscle weight analysis confirmed these findings ( $p$ $<0.01$, Fig. 2 B, table 2).

Next, by H\&E staining, we identified areas containing tumor cells in C26-injected muscles (Fig. 3 A and 4 A). Regions containing tumor cells were characterized by a high number of cells per area. Tumor cells had larger nuclei compared to the myonuclei and mononuclear cells observed in the tumor areas, as
$A$
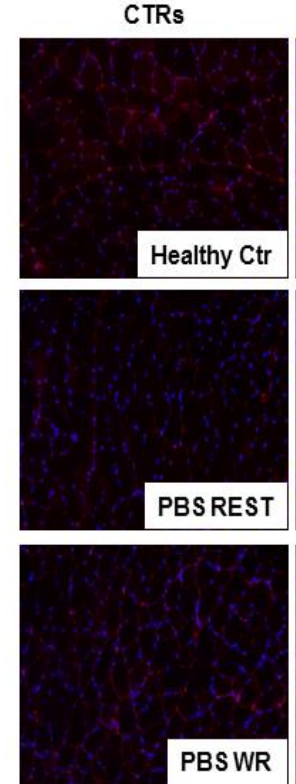

TAREST
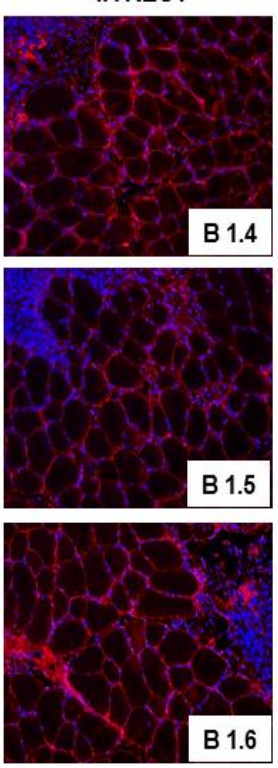

TA WR
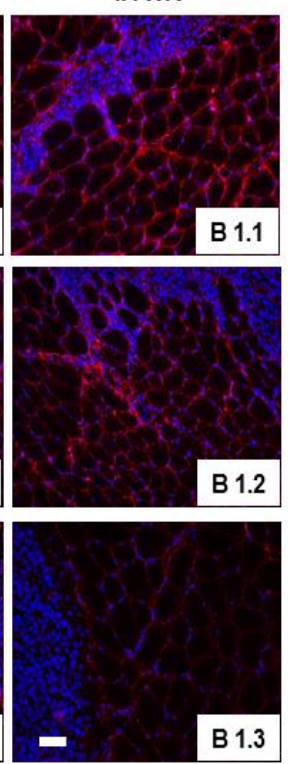

B

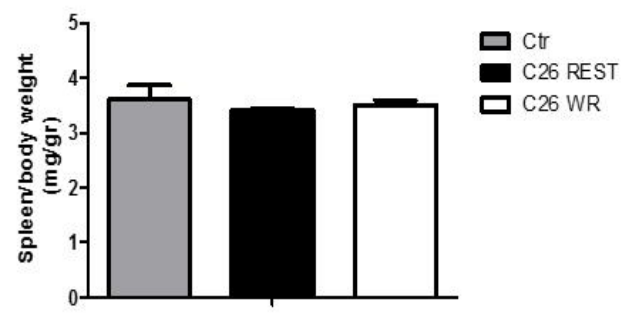

Fig 6. Inflammatory background. (A) Representative images of local inflammation in control and tumor cellsinjected muscles. Red, mouse IgG, blue Hoechst. Bar = $100 \mu \mathrm{m}$. (B) Spleen weight analysis between control, C26 REST and C26 WR mice. Error bars are shown as means \pm SEM of 3 independent experiments. 


\section{Physical activity counteracts tumor cell growth in muscle}

Eur J Transl Myol 26 (2): 67-75

shown in figure $3 \mathrm{~A}$. Moreover, the staining for F4/80 revealed only a limited amount of macrophage cells within the tumor areas (Fig. 3 B). To investigate the nature of the differences in size and weight of injected muscles between non-exercised and running mice, we analyzed the tumor areas developed in muscles (Fig. 4 A). Specifically, cross-sectional area (CSA) analysis of C26-injected muscles showed that tumor cells expanded at the site of injection. No single cells were found far from the side of injection (Fig. $3 \mathrm{~A}$ and $4 \mathrm{~A}$ ).

Morphometric analysis showed that in C26-injected muscles from sedentary mice the tumor cells represent $7.9 \% \pm 1.2$ of the total muscle CSA, while in muscles from the C26-injected running group the average of tumor cells-covered area was $5.1 \% \pm 1.3 / \mathrm{CSA}$ (Fig. 4 B). These results indicate a trend of reduced tumor growth in the running mice. Since tumor cells are characterized by high proliferative rates, we further investigated the effects of physical activity on tumor cell growth by analyzing the cells expressing Ki-67, a known marker of cellular proliferation (Fig. $5 \mathrm{~A}$ and $\mathrm{B}$ ). ${ }^{24}$ This approach allowed us to better determine the extent and the impact of tumor cells growth in the muscle tissue, without the contribution of other cells (e.g. inflammatory infiltrate), which were limited compared to the number of tumor cells in C26-injected muscles (Fig. 3 B). Quantitative analysis of Ki-67 positive cells from tumor areas showed a significant reduction of tumor cell proliferation in muscles of running mice compared to those derived from sedentary mice ( $<<0.05$, Fig. 5 B).

Because tumor development is normally associated with an inflammatory response in the target tissue, we also analyzed the inflammatory state of injected muscles (Fig. 6). Immunoglobulin staining revealed a high inflammatory background in C26-injected muscles, while a basal level of IgG was found in both PBSinjected and healthy control muscles (Fig. 6 A). These observations indicate that tumor cells induced local inflammation. Next, we measured the weight of the spleen, an organ sensitive to chronic inflammatory conditions including cancer. Quantitative analysis showed a comparable weight of the spleen between C26injected and non-injected mice (Fig. 6 B). These data, together with the analysis of body weight of nonexercised tumor-injected mice, which showed no changes in body weight between the day of tumor cells injection and the end of experiment (Table 2), confirmed that tumor cells mediated at local level the muscle changes without any further contribution due to the presence of a chronic systemic inflammation.

\section{Discussion}

A general consensus has been reached in the last years concerning the beneficial effects of physical exercise in the prevention, management and treatment of chronic degenerative conditions, ${ }^{6}$ including cancer. ${ }^{6,11,12}$ Currently, public health offices recommend regular physical exercise for a healthy lifestyle. ${ }^{6}$
In cancer epidemiology, physical activity is associated with a reduced incidence, a better prognosis, an increased responsiveness to therapy and a general improvement in patients' quality of life. ${ }^{11}$ These evidences result from clinical studies performed on specific tumor types, including breast, colon and pancreatic cancers. ${ }^{11}$ The effects of physical activity on tumor growth have long been debated. ${ }^{25,26}$ Interestingly, a recent study showed that voluntary running exercise leads to a remodeling of immune cells colonizing the tumor microenvironment of tumortransplanted mice. ${ }^{17}$ In particular, researchers showed that physical activity negatively affects tumor growth by increasing the recruitment of NK cells within the tumor mass. ${ }^{17}$

Skeletal muscle tissue is the main target of the pathophysiological changes occurring both in early, ${ }^{27-29}$ and in advanced stages of cancer progression, such as loss of muscle mass and function. ${ }^{30,31}$ The elevated systemic inflammation originating from the tumor is a pivotal mediator of muscle wasting observed in cancer patients. $^{2}$ The inflammation also affects other organs and tissues (e.g. fat tissue $)^{3}$, a condition known as cancer cachexia syndrome. Thus, according to data reported in the literature, there is a strong correlation between tumor progression and immune response. ${ }^{32-34}$ In this picture, the elevated inflammatory background can mask direct beneficial effects of physical activity on tumor cells growth and the molecular mechanisms involved.

Here, we analyzed the direct effects of physical activity on tumor cell growth in a chronic systemic inflammation-independent context. Specifically, we adopted voluntary running as a form of exercise compatible with advanced stages of tumor progression, as emerged by clinical studies performed in cancer patients. ${ }^{15} \mathrm{We}$ injected $\mathrm{C} 26$ colon carcinoma cells in TA muscles of BALB/c mice, to directly determine the effects of muscle contraction on their growth.

Muscle is the most represented and metabolically active tissue in the body, and its capillary bed is certainly extremely extended. Thus, it could theoretically represent a suitable site for tumor development. However, clinical reports only rarely document events of tumor metastasis occurring in skeletal muscles. So far, tumor metastasis in muscle tissue have been reported only in a few cases of laryngeal squamous carcinoma, ${ }^{35-37}$ lung cancer, ${ }^{38}$ papillary thyroid cancer $^{39}$ bladder cancer, ${ }^{40}$ and gastrointestinal stromal tumor. ${ }^{41,42}$

Our data show that muscles injected with C26 cells develop tumor cells expansion after 4 days of cell implantation, suggesting that muscle tissue per se is a permissive environment for tumor cell growth. Histological analyses revealed that a total of $\sim 15$ hours of voluntary wheel running (table 1), which is considered a low-intensity ${ }^{43,44}$ and aerobic ${ }^{12}$ exercise, accumulated over a period of 4 days leads to a 


\section{Physical activity counteracts tumor cell growth in muscle}

Eur J Transl Myol 26 (2): 67-75

significant reduction of tumor cell growth compared with muscles from sedentary mice. Interestingly, we injected tumor cells in muscles of healthy mice and samples were analyzed 4 days later, so the observed tumor cell growth was independent of the systemic inflammation usually reported after longer periods in tumor bearing mouse models. This aspect was confirmed by weight analysis of the spleen, that revealed no differences between healthy and C26 muscle-injected mice. On the contrary, an increased local inflammatory background was observed in C26-injected muscles from both sedentary and running mice, compared with noninjected and PBS-injected muscles.

Overall, our results indicate that, although the muscle microenvironment is generally unfavorable for growth of metastatic cancer cells, its refractoriness can be modulated by exercise.

The observations of this study confirmed that physical activity acts as negative regulator for tumor cells growth in skeletal muscle tissue. However, further analyses are needed to better investigate the relationship between tumor-mediated local inflammation, tumor dissemination and physical activity. Whether the inflammatory, reparative and myoregenerative responses that may occur in muscle during wheel running ${ }^{27-29,45-47}$ may have direct effects on the metastatic processes remain to be investigated.

\section{Contributions}

EB conceived and supervised the project. $\mathrm{EB}$ and $\mathrm{CH}$ performed the experiments and optimized the methodology. TV, KK and SDS helped the elaboration of the methodology and the prototype characterization. $\mathrm{EB}$ wrote the manuscript and $\mathrm{PH}$ helped in revising it.

\section{Acknowledgements}

E. Berardi is supported by Research Fund - Flanders (FWO) $(12 \mathrm{D} 2816 \mathrm{~N})$, and $(1525315 \mathrm{~N})$. T. Vandoorne is supported by FWO-fellowship strategic basic research (1S60116N). The authors would like to thank Monique Ramaekers for her technical support and Professor Ugo Carraro for critical discussion.

\section{Conflict of Interest}

The authors declare no potential conflict of interests.

\section{Corresponding Author}

Emanuele Berardi, Exercise Physiology Research Group, Department of Kinesiology, KU Leuven, Tervuursevest

101 B-3001, Leuven, Belgium.

Tel. +3216372662

E-mail: emanuele.berardi@med.kuleuven.be

E-mails of coAuthors

Charlotte Hiroux: charlotte.hiroux@kuleuven.be

Tijs Vandoorne: tijs.vandoorne@kuleuven.be

Katrien Koppo: katrien.koppo@kuleuven.be

Stefan De Smet: stefan.desmet@kuleuven.be

Peter Hespel: peter.hespel@kuleuven.be

\section{References}

1. Fearon K, Strasser F, Anker SD, et al. Definition and classification of cancer cachexia: an international consensus. Lancet Oncol 2011;12:489-95.

2. Argiles JM, Busquets S, Stemmler B, et al. Cancer cachexia: understanding the molecular basis. Nat Rev Cancer 2014;14:754-62.

3. Fearon KC: Cancer cachexia and fat-muscle physiology. N Engl J Med 2011;365:565-7.

4. Vaughan VC, Martin P, Lewandowski PA. Cancer cachexia: impact, mechanisms and emerging treatments. J Cachexia Sarcopenia Muscle 2013;4:95-109.

5. Tisdale MJ. Mechanisms of cancer cachexia. Physiol Rev 2009;89:381-410.

6. Egan B, Zierath JR. Exercise metabolism and the molecular regulation of skeletal muscle adaptation. Cell Metab 2013;17:162-184.

7. Pekmezi DW, Demark-Wahnefried W. Updated evidence in support of diet and exercise interventions in cancer survivors. Acta Oncol 2011;50:167-78.

8. Speck RM, Courneya KS, Masse LC, et al. An update of controlled physical activity trials in cancer survivors: a systematic review and metaanalysis. J Cancer Surviv 2010;4:87-100.

9. Coletti D, Aulino P, Pigna E, et al. Spontaneous Physical Activity Downregulates Pax7 in Cancer Cachexia. Stem Cells Int 2016; 2016:6729268.

10. Puetz TW, Herring MP. Differential effects of exercise on cancer-related fatigue during and following treatment: a meta-analysis. Am J Prev Med 2012; 43:e1-24.

11. Leitzmann M, Powers H, Anderson AS, et al. European Code against Cancer 4th Edition: Physical activity and cancer. Cancer Epidemiol 2015; 39 Suppl 1:S46-55.

12. Gould DW, Lahart I, Carmichael AR, et al. Cancer cachexia prevention via physical exercise: molecular mechanisms. J Cachexia Sarcopenia Muscle 2013;4:111-24.

13. Mishra SI, Scherer RW, Geigle PM, et al. Exercise interventions on health-related quality of life for cancer survivors. Cochrane Database Syst Rev 2012, 8:CD007566.

14. Swisher AK, Abraham J, Bonner D, et al. Exercise and dietary advice intervention for survivors of triple-negative breast cancer: effects on body fat, physical function, quality of life, and adipokine profile. Support Care Cancer 2015;23:2995-3003.

15. Stene GB, Helbostad JL, Balstad TR, et al. Effect of physical exercise on muscle mass and strength in cancer patients during treatment - a systematic review. Crit Rev Oncol Hematol 2013;88:573-93.

16. Pin F, Busquets S, Toledo M, et al. Combination of exercise training and erythropoietin prevents 


\section{Physical activity counteracts tumor cell growth in muscle}

Eur J Transl Myol 26 (2): 67-75

cancer-induced muscle alterations. Oncotarget 2015;6:43202-15.

17. Pedersen L, Idorn M, Olofsson GH, et al. Voluntary Running Suppresses Tumor Growth through Epinephrine- and IL-6-Dependent NK Cell Mobilization and Redistribution. Cell Metab 2016;23:554-52.

18. Argiles JM, Stemmler B, Lopez-Soriano FJ, et al. Nonmuscle Tissues Contribution to Cancer Cachexia. Mediators Inflamm 2015;2015:182872.

19. Parlakian A, Gomaa I, Solly S, et al. Skeletal Muscle Phenotypically Converts and Selectively Inhibits Metastatic Cells in Mice. Plos One 2010;5:e9299.

20. Steinbach S, Yuh EL, Burbelko M, et al. Detection of Micrometastases of Squamous Cell Carcinoma Tumor Cells in Muscle Tissue. Anticancer Res 2013;33:5213-21.

21. Tzukerman M, Rosenberg T, Ravel Y, et al. An experimental platform for studying growth and invasiveness of tumor cells within teratomas derived from human embryonic stem cells. P Natl Acad Sci USA 2003;100:13507-12.

22. Kilkenny C, Browne WJ, Cuthill IC, et al. Improving bioscience research reporting: the ARRIVE guidelines for reporting animal research, PLoS Biol 2010;8:e1000412.

23. Coletti D, Berardi E, Aulino P, et al. Substrains of inbred mice differ in their physical activity as a behavior. Scientific World Journal 2013;2013:237260.

24. Schluter C, Duchrow M, Wohlenberg C, et al. The cell proliferation-associated antigen of antibody $\mathrm{Ki}$ 67: a very large, ubiquitous nuclear protein with numerous repeated elements, representing a new kind of cell cycle-maintaining proteins. J Cell Biol 1993;123:513-22.

25. Cao L, Liu X, Lin EJ, et al. Environmental and genetic activation of a brain-adipocyte BDNF/leptin axis causes cancer remission and inhibition. Cell 2010;142:52-64.

26. Westwood JA, Darcy PK, Kershaw MH. Environmental enrichment does not impact on tumor growth in mice. F1000Res 2013;2:140.

27. Zampieri S, Doria A, Adami N, et al. Subclinical myopathy in patients affected with newly diagnosed colorectal cancer at clinical onset of disease: evidence from skeletal muscle biopsies. Neurol Res 2010;32:20-5.

28. Zampieri S, Valente $\mathrm{M}$, Adami $\mathrm{N}$, et al. Polymyositis, dermatomyositis and malignancy: a further intriguing link. Autoimmun Rev 2010;9:449-53.

29. Adami N. Serum and tissue markers of myopathy in patients with colorectal cancer. European Journal of Translational Myology 2012;22:1 125-46. doi: 10.4081/ejtm.2012.1796.
30. Aulino P, Berardi E, Cardillo VM, et al. Molecular, cellular and physiological characterization of the cancer cachexia-inducing C26 colon carcinoma in mouse. BMC Cancer 2010;10:363.

31. Berardi E, Aulino P, Murfuni I, et al. Skeletal muscle is enriched in hematopoietic stem cells and not inflammatory cells in cachectic mice. Neurol Res 2008;30:160-9.

32. Grivennikov SI, Greten FR, Karin M. Immunity, inflammation, and cancer. Cell 2010;140:883-99.

33. Imai K, Matsuyama S, Miyake S, et al. Natural cytotoxic activity of peripheral-blood lymphocytes and cancer incidence: an 11-year follow-up study of a general population. Lancet 2000;356:1795-9

34. Vivier E, Ugolini S, Blaise D, et al. Targeting natural killer cells and natural killer $\mathrm{T}$ cells in cancer. Nat Rev Immunol 2012;12:239-52

35. Kulahci Y, Zor F, Onguru O, et al. Distant muscular (rectus femoris) metastasis from laryngeal squamous cell carcinoma. J Laryngol Otol 2009;123:1381-3.

36. Lucas Z, Mukherjee A, Chia S, et al. Metastasis of laryngeal squamous cell carcinoma to bilateral thigh muscles. Case Rep Oncol Med 2014;2014:424568.

37. Marioni G, Blandamura S, Calgaro $\mathrm{N}$, et al. Distant muscular (gluteus maximus muscle) metastasis from laryngeal squamous cell carcinoma. Acta Otolaryngol 2005; 125:678-82.

38. Tezcan Y, Koc M. Muscle metastasis from nonsmall cell lung cancer: two cases and literature review. Acta Clin Belg 2014;69:302-4.

39. Yang J, Li LF, Zhang XM, et al. Unusual synchronous skeletal muscle and lung metastasis in papillary thyroid cancer: A case report and review of the literature. Oncol Lett 2015;9:72730.

40. Katafigiotis I, Athanasiou A, Levis PK, et al. Metastasis to sartorius muscle from a muscle invasive bladder cancer. Case Rep Med 2014;2014:524757.

41. Cichowitz A, Thomson BN, Choong PF. GIST metastasis to adductor longus muscle. ANZ J Surg 2011;81:490-1.

42. Pasku D, Karantanas A, Giannikaki E, et al. Bilateral gluteal metastases from a misdiagnosed intrapelvic gastrointestinal stromal tumor. World J Surg Oncol 2008;6:139.

43. Allen DL, Harrison BC, Maass A, et al. Cardiac and skeletal muscle adaptations to voluntary wheel running in the mouse. J Appl Physiol (1985) 2001;90:1900-8.

44. Holick CN, Newcomb PA, Trentham-Dietz A, et al. Physical activity and survival after diagnosis of invasive breast cancer, Cancer Epidemiol Biomarkers Prev 2008;17:379-86. 


\section{Physical activity counteracts tumor cell growth in muscle}

Eur J Transl Myol 26 (2): 67-75

45. Carraro U, Franceschi C. Apoptosis of skeletal and cardiac muscles and physical exercise, Aging (Milano) 1997;9:19-34.

46. Podhorska-Okolow M, Sandri M, Zampieri S, et al. Apoptosis of myofibres and satellite cells: exerciseinduced damage in skeletal muscle of the mouse. Neuropathol Appl Neurobiol 1998;24:518-531.
47 Pigna E, Berardi E, Aulino P. et al. Aerobic Exercise and Pharmacological Treatments Counteract Cachexia by Modulating Autophagy in Colon Cancer. Scientific Reports 2016; 31;6:26991. 